\title{
CONDITIONS FOR THE SOLUBILITY OF AN ELLIPTIC DIFFERENCE EQUATION AS AN INITIAL VALUE PROBLEM*
}

\author{
$\mathrm{BY}$ \\ K. J. HARKER AND J. LLACER \\ Microwave Laboratory, W. W. Hansen Laboratories of Physics, Stanford University
}

1. Introduction. It is understood, in general, that the solution of elliptic partial differential equations as initial value problems by the method of finite differences is unsuccessful due to lack of convergence and to instability. However, careful analysis of a representative problem points out that, when the initial values are analytic, useful regions of convergence and conditions to assure stability can be found. The theoretical analysis to be undertaken follows basically the ideas developed by Dahlquist[1]. Laplace's equation in two dimensions was chosen as a representative elliptic partial differential equation.

2. Two-dimensional problem in rectangular coordinates. a) Determination of region of convergence. Consider Laplace's equation in two dimensions, with rectangular coordinates $x$ and $t$ :

$$
V_{x x}+V_{t t}=0 .
$$

If we define

$$
u=V_{x}, \quad v=V_{t},
$$

we obtain the Cauchy-Riemann equations:

$$
u_{t}=v_{x}, \quad u_{x}=-v_{t} .
$$

Further, if we define a complex function of $x$ and $t$ as

$$
W=u \pm i v,
$$

we obtain

$$
W_{t}=u_{t} \pm i v_{t},
$$

and by application of the Cauchy-Riemann equations as given above, we obtain finally the equation

$$
W_{t}=\mp i W_{x},
$$

which is equivalent to Eq. (1) in a complex form. Clearly any solution of Eq. (2) can be readily transformed into a solution of Eq. (1) by simple integration of the real and/or imaginary parts of $W$.

For the solution of this problem by the method of finite differences, consider the triangular mesh given by Fig. 1.

Equation (2) can be approximated by the difference equation

* Received June 26, 1962; revised manuscript received April 17, 1963. The work reported in this paper was sponsored by the Air Force Systems Command, Rome Air Development Center, Griffiss Air Force Base, New York, under Contract AF 30(602)-2575. 


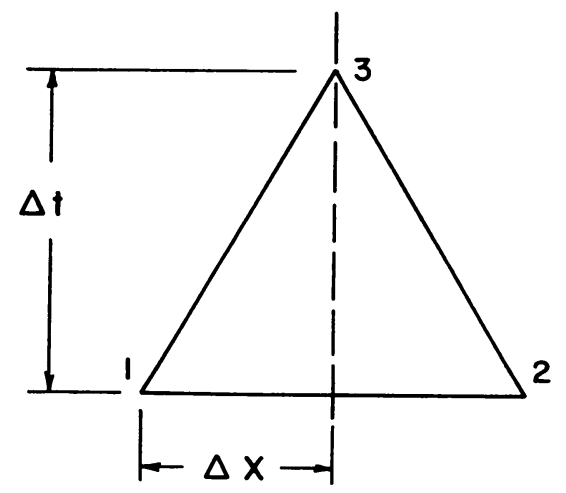

Fig. 1. Triangular mesh system

$$
\frac{W_{3}-\left(W_{1}+W_{2}\right) / 2}{\Delta t}=\mp i \frac{\left(W_{2}-W_{1}\right)}{2 \Delta x} .
$$

Particular solutions to Eq. (3) can be written in the form

$$
W(x, t)=e^{(\gamma t+\lambda x)},
$$

or

$$
W(x, t)=e^{(\gamma t+i \lambda x)},
$$

where $\gamma$ and $\lambda$ are real. With these solutions, Eq. (3) becomes

$$
\frac{e^{\lambda \Delta t}-\cosh \lambda \Delta x}{\Delta t}=\mp i \frac{\sinh \lambda \Delta x}{\Delta x}
$$

or

$$
\frac{e^{\gamma \Delta t}-\cos \lambda \Delta x}{\Delta t}= \pm \frac{\sin \lambda \Delta x}{\Delta x}
$$

respectively.

By solving for $\gamma \Delta t$ and substituting into (4a) and (4b), we are able to eliminate $\gamma$ and rewrite the particular solutions in the new forms:

$$
W(x, t)=\left(\cosh \lambda \Delta x \mp i \mu^{-1} \sinh \lambda \Delta x\right)^{t / \Delta t} e^{\lambda x},
$$

or

$$
W(x, t)=\left(\cos \lambda \Delta x \pm \mu^{-1} \sin \lambda \Delta x\right)^{t / \Delta t} e^{i \lambda x},
$$

where $\mu=\Delta x / \Delta t$.

Consider now initial values $W(x, 0)$ given along the $x$-axis for the solution of a particular problem. In using Eq. (3) to find the value of $W(x, t)$ at any point off the $x$ axis, we can use the fact that this equation is linear. We are able, therefore, to define a linear operator $\alpha_{t}$ such that

$$
\alpha_{t}[W(x, 0)]=W(x, \imath)
$$

Moreover, we can find representations for $\alpha_{\imath}$ by considering Eqs. (5): For $t=0$, (5a) 
becomes

$$
W(x, 0)=e^{\lambda x}
$$

and $(5 \mathrm{~b})$ becomes

$$
W(x, 0)=e^{i \lambda x} .
$$

Operating with $\alpha_{t}$ on these initial values, and comparing with Eqs. (5) we see that

$$
\alpha_{t}=\left(\cosh \lambda \Delta x \mp i \mu^{-1} \sinh \lambda \Delta x\right)^{t / \Delta t},
$$

or

$$
\alpha_{t}=\left(\cos \lambda \Delta x \pm \mu^{-1} \sin \lambda \Delta x\right)^{t / \Delta t},
$$

respectively.

Keeping these results in mind, let us turn our attention to a problem with initial values

$$
W(x, 0)=\frac{1}{x-\zeta},
$$

where $x$ is real and $\zeta=\zeta^{\prime}+i \zeta^{\prime \prime}$.

From the theory of Laplace and Fourier transforms, we can represent the initial values of Eq. (7) as integrals, with appropriate restrictions on $\zeta$ :

$$
\begin{aligned}
& \frac{1}{x-\zeta}=-\int_{0}^{\infty} e^{-\lambda \zeta} e^{\lambda x} d \lambda \quad \text { for } \quad \zeta^{\prime}>x \\
& =\int_{0}^{\infty} e^{\lambda \zeta} e^{-\lambda x} d \lambda \quad \text { for } \quad \zeta^{\prime}<x \\
& =-i \int_{0}^{\infty} e^{-i \lambda \zeta} e^{i \lambda x} d \lambda \text { for } \zeta^{\prime \prime}<0 \\
& =i \int_{0}^{\infty} e^{i \lambda \zeta} e^{-i \lambda x} d \lambda \quad \text { for } \quad \zeta^{\prime \prime}>0 .
\end{aligned}
$$

If we now operate with $\alpha_{t}$, as given by Eqs. (6), on Eqs. (8) through (11), and we use the linearity of the operator to bring it under the integral sign, we obtain the four corresponding equations:

$$
\begin{aligned}
W(x, t)=\alpha_{t} & \left(\frac{1}{x-\zeta}\right) \\
& =-\int_{0}^{\infty} e^{-\lambda \zeta} e^{\lambda x}\left(\cosh \lambda \Delta x \mp i \mu^{-1} \sinh \lambda \Delta x\right)^{t / \Delta t} d \lambda \text { for } \zeta^{\prime}>\cdot x, \\
& =\int_{0}^{\infty} e^{\lambda \zeta} e^{-\lambda x}\left(\cosh \lambda \Delta x \mp i \mu^{-1} \sinh \lambda \Delta x\right)^{t / \Delta t} d \lambda \quad \text { for } \quad \zeta^{\prime}<x, \\
& =-i \int_{0}^{\infty} e^{-i \lambda \zeta} e^{i \lambda x}\left(\cos \lambda \Delta x \pm \mu^{-1} \sin \lambda \Delta x\right)^{t / \Delta t} d \lambda \text { for } \quad \zeta^{\prime \prime}<0, \\
& =i \int_{1}^{\infty} e^{i \lambda \zeta} e^{-i \lambda x}\left(\cos \lambda \Delta x \pm \mu^{-1} \sin \lambda \Delta x\right)^{t / \Delta t} d \lambda \quad \text { for } \quad \zeta^{\prime \prime}>0 .
\end{aligned}
$$


A sufficient condition for the integrals in (12) through (15) to converge uniformly with respect to $\Delta t$ for constant $\mu$, is that the absolute value of its integrand be equal to or smaller than some function $G(\lambda)$, independent of $\Delta t$, such that $\int_{0}^{\infty} G(\lambda) d \lambda$ converges [2].

For Eq. (12), we write

$$
\left|e^{-\lambda \xi} e^{\lambda x}\left(\cosh \lambda \Delta x \mp i \mu^{-1} \sinh \lambda \Delta x\right)^{t / \Delta t}\right| \leq \eta,
$$

where

$$
\eta=e^{-\lambda \xi^{\prime}} e^{\lambda x}\left(\cosh ^{2} \lambda \Delta x+\mu^{-2} \sinh ^{2} \lambda \Delta x\right)^{t / 2 \Delta \prime} .
$$

Rearrangement of $\eta$ leads to the form

$$
\eta=e^{-\lambda \zeta^{\prime}} e^{\lambda x} e^{\lambda \mu t}\left\{\left[\left(\frac{1+e^{-2 \lambda \Delta x}}{2}\right)^{2}\left(1+\mu^{-2} \tanh ^{2} \lambda \Lambda x\right)\right]^{1 / \lambda \Delta x}\right\}^{t \lambda \mu / 2} .
$$

By letting $\phi(\mu)$ stand for one-half the natural logarithm of the maximum value of the expression appearing between braces, as a function of $\mu$, we then have

$$
\left|e^{-\lambda \xi} e^{\lambda x}\left(\cosh \lambda \Delta x \mp i \mu^{-1} \sinh \lambda \Delta x\right)^{t / \Delta t}\right| \leq e^{-\lambda \xi^{\prime}} e^{\lambda x} e^{\lambda \mu t} e^{t \lambda \mu \phi}=G(\lambda) .
$$

Then, $\int_{0}^{\infty} G(\lambda) d \lambda$ will remain bounded if

$$
-\zeta^{\prime}+x+\mu t(1+\phi)<0,
$$

or

$$
\zeta^{\prime}>x+\mu t(1+\phi) \text {. }
$$

The function $\phi(\mu)$ is plotted in Fig. 2. Similar treatment of Eq. (13) yields

$$
\zeta^{\prime}<x-\mu t(1+\phi) \text {. }
$$

For Eq. (14), by observing that $\left|\cos \lambda \Delta x \pm \mu^{-1} \sin \lambda \Delta x\right|^{t / \Delta t}=\left\{\left|\cos \lambda \Delta x \pm \mu^{-1} \sin \lambda \Delta x\right|^{1 / \lambda \Delta x}\right\}^{t \lambda \mu}$,

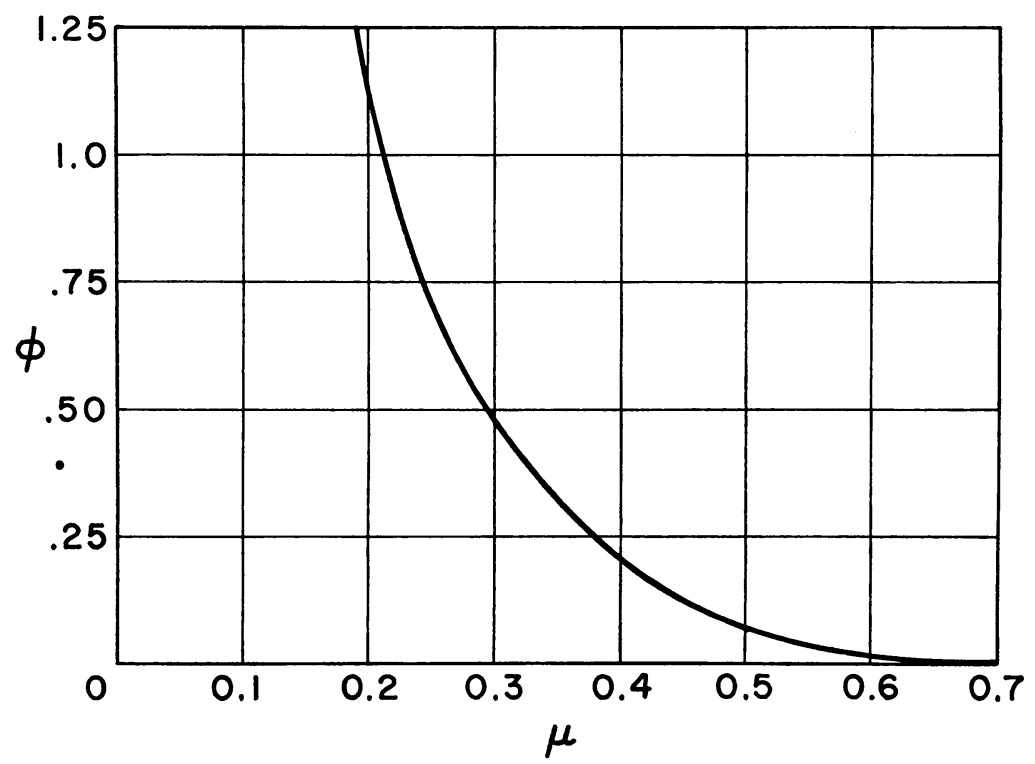

FIg. 2. Variation of $\phi$ as a function of the mesh ratio, $\mu$ 
and that the maximum value of the expression between brackets is $e^{1 / \mu}$, we can write

$$
\left|e^{-i \lambda \zeta} e^{i \lambda x}\left(\cos \lambda \Delta x \pm \mu^{-1} \sin \lambda \Delta x\right)^{t / \Delta t}\right| \leq e^{\lambda \zeta^{\prime \prime}} e^{\lambda t}=G(\lambda)
$$

and $\int_{0}^{\infty} G(\lambda) d \lambda$ will remain bounded if

$$
\xi^{\prime \prime}+t<0
$$

or

$$
\zeta^{\prime \prime}<-t
$$

Similarly, from (15) we obtain

$$
\zeta^{\prime \prime}>t \text {. }
$$

Inequalities (16) through (19) give, independently, sufficient conditions under which a solution to Eq. (3) can be found, given initial conditions of the form $W(x, 0)=1 /(x-\zeta)$. They define a rectangle in the $\left(\zeta^{\prime}, \zeta^{\prime \prime}\right)$ plane, for a given $(x, t)$, outside of which $\left(\zeta^{\prime} \zeta^{\prime \prime}\right)$ must lie if the solution $\alpha_{t}[1 /(x-\zeta)]$ is to converge.

Next, these conditions are generalized to the whole class of analytic functions by the use of the Cauchy integral formula. If $f$ is analytic in and on a contour $C$, including a portion of the real axis, then for any point $x$ on the real axis inside $C$, we have

$$
f(x)=\frac{1}{2 \pi i} \int_{C} \frac{f(\zeta)}{(x-\zeta)} d \zeta
$$

Furthermore, since $\alpha_{t}$ is a linear operator and linear operations can be performed under the integral sign, we have

$$
\alpha_{t}(f)=\frac{1}{2 \pi i} \int_{C} f(\zeta) \alpha_{t}[1 /(x-\zeta)] d \zeta
$$

If $f(x)$ represents the value of an analytic function along the initial boundary line $t=0$, $\alpha_{t}(f)$ will represent the solution of Eq. (3) at any $(x, t)$ with those initial values. However, the right hand side of Eq. (20) imposes two conditions: (1) $f(\zeta)$ must be analytic in and on $C$, and (2) $\alpha_{t}[1 /(x-\zeta)]$ must converge uniformly with respect to $\Delta t$ on $C$. These two requirements are met if the points $\left(x_{i}, t_{i}\right)$ corresponding to the singularities $\left(x_{i}+i t_{i}\right)$ of $f$ fall outside the rectangle defined by inequalities (16) through (19). If a singularity of $f$ lies at $\left(x_{1}+i t_{1}\right)$, we can rewrite inequalities (16) through (19) as follows:

$$
\left.\begin{array}{c}
x_{1}>x+\mu t(1+\phi) \\
x_{1}<x-\mu t(1+\phi)
\end{array}\right\}
$$

Inequalities (21) and (22) define two regions in which $\alpha_{t}(f)$ converges. The superposition of these two regions gives, then, the region in the real $(x, t)$ plane in which a solution to Eq. (3), subject to initial values $f$, will be obtainable (Fig. 3).

We can then state: Let $f(x)$ be the value of an analytic function at the line $t=0$ in the $(x, t)$ plane. A sufficient condition for obtaining a solution to Laplace's equation, subject to the initial values given by $f(x)$, by the method of finite differences, is that we do not operate inside the "shadows" of a singularity $\left(x_{1}+i t_{1}\right)$ of $f$. These "shadows" 


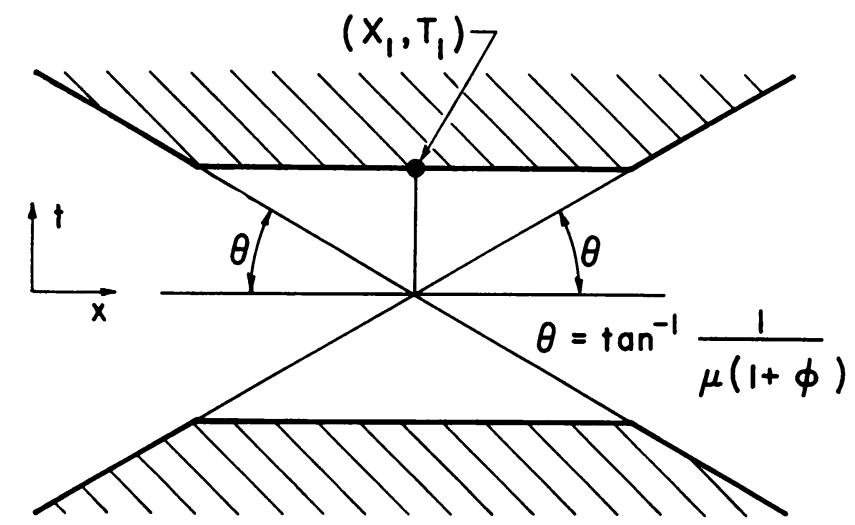

FIG. 3. "Shadows" of a singularity at $\left(x_{1}+i t_{1}\right)$. In the region exterior to the shadows it is proven that convergence is obtained.

are defined by the inequalities

$$
\begin{aligned}
& |t|>\left|\frac{x_{1}-x}{\mu(1+\phi)}\right| \\
& |t|>\left|t_{1}\right|
\end{aligned}
$$

where

$$
\mu=\Delta x / \Delta t
$$

and $\phi$ is given by Fig. 2 .

b) Conditions for Stability. In improper problems like the one under consideration, one can, in general, expect instability, i.e., lack of continuous dependence of the solution on the initial data. However, F. John [3] shows that we can expect Hölder continuity of the solution on intial values for a more general elliptic Cauchy problem, with suitable restrictions, provided that the initial values are analytic. Since our problem falls within those restrictions, we are assured of the stability of the solution found in the regions of convergence indicated above, assuming that our initial values are analytic.

In practice, however, as is the case in the use of digital computers, we are limited in the representation of initial values to a certain number of digits. This round-off error destroys analyticity and, in general, the inherent instability of improper problems prevents us from finding a solution. In spite of this fact, a study of error growth leads to establishing a relationship between mesh size, number of digits used in the computation, and error growth which allows us to find a stable, convergent solution to the problem under discussion.

Consider Laplace's equation, $V_{x x}+V_{t}=0$, broken down into two first-order differential equations:

$$
\begin{aligned}
& u_{t}=v_{x} \\
& u_{x}=-v_{t},
\end{aligned}
$$

where $u=V_{x}$ and $v=V_{t}$. To solve this system of equations using the triangular mesh given by Fig. 1, we form the difference equations

$$
\frac{u_{3}-\left(u_{1}+u_{2}\right) / 2}{\Delta t}=\frac{v_{2}-v_{1}}{2 \Delta x},
$$




$$
\frac{v_{3}-\left(v_{1}+v_{2}\right) / 2}{\Delta t}=\frac{u_{2}-u_{1}}{2 \Delta x}
$$

From known values of $v$, for example, we can determine $V$ by the equation

$$
\frac{V_{3}-\left(V_{1}+V_{2}\right) / 2}{\Delta t}=\frac{v_{1}+v_{2}}{2}
$$

Because of linearity, we can use Eqs. (24) through (26) to determine the rate of growth of round-off errors. Let $u, v$, and $V$ stand for these errors and assume solutions to the difference equations of the form

$$
u=A e^{\alpha t+i \beta x} ; \quad v=B e^{\alpha t+i \beta x} ; \quad V=C e^{\alpha t+i \beta x} .
$$

By placing these solutions into (24) through (26), and reducing, we obtain the following set of equations:

$$
\left(\begin{array}{ccc}
e^{\alpha \Delta t}-\cos \beta \Delta x & -i \sin \beta \Delta x & 0 \\
i \sin \beta \Delta x & e^{\alpha \Delta t}-\cos \beta \Delta x & 0 \\
0 & -\Delta t \cos \beta \Delta x & e^{\alpha \Delta t}-\cos \beta \Delta x
\end{array}\right)\left(\begin{array}{l}
A \\
B \\
C
\end{array}\right)=0 .
$$

By setting the determinant of the matrix equal to zero, we obtain

$$
\left(e^{\alpha \Delta t}-\cos \beta \Delta x\right)^{2}-\sin ^{2} \beta \Delta x=0 .
$$

It is to be noted that the presence of Eq. (26) has no effect on the condition given by Eq. (28). This is to be expected, since Eq. (26) has a completely stable solution. By reducing Eq. (28), we obtain

$$
e^{\alpha \Delta t}=\sqrt{1 \pm \sin 2 \beta \Delta x} .
$$

This equation relates the constant $\alpha$, controlling the growth of errors in the $t$-direction, with $\beta=2 \pi / \lambda$, where $\lambda$ is the wavelength of the particular component of the error solution under analysis.

To find the component with largest contribution to error growth, we maximize Eq. (29) by requiring that $2 \beta \Delta x=(2 n+1) \pi / 2$, or

$$
\lambda=\frac{8 \Delta x}{2 n+1} \text {. }
$$

The only value of $\lambda$ integral in $\Delta x$ is given $\lambda=8 \Delta x$, corresponding to $n=0$. The associated value of $\alpha$ is given by

$$
\alpha=\frac{\ln 2}{2 \Delta t}
$$

The magnitude of the $\lambda=8 \Delta x$ component of the errors introduced at the base line will be of the order of the least significant digit used to represent the initial values and perform the computations.

This, together with Eq. (30), gives a practical rule for determining mesh size and number of digits necessary for the computation in order to obtain a prescribed relative error at a certain value of $t$. For a mesh system different from the one of Fig. 1, an analysis parallel to the present one can be carried out. 


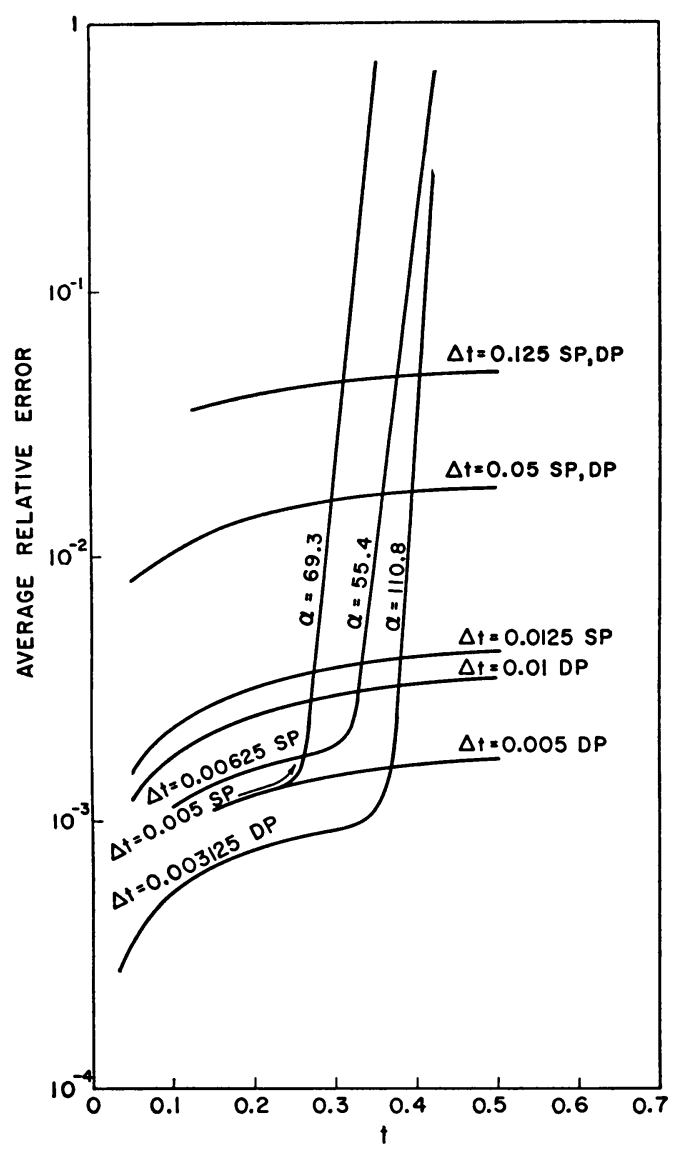

FIg. 4. Average relative error as a function of $t$ for $\mu=1$.

3. Numerical results of a particular problem. The problem of solving Laplace's equation in two dimensions, subject to initial values

$$
V(x, 0)=\ln (1+x) ; \quad V_{t}(x, 0)=0 ; \quad 0 \leq x \leq 1,
$$

was undertaken. We solve the pair of first order equations (23) with the equivalent initial conditions

$$
u(x, 0)=\frac{1}{1+x} ; \quad v(x, 0)=0 ; \quad 0 \leq x \leq 1
$$

Let $u+i v=(1+x-i t)^{-1}$; we then have

$$
u=\frac{1+x}{(1+x)^{2}+t^{2}}, \quad v=\frac{t}{(1+x)^{2}+t^{2}} .
$$

Clearly, Eqs. (32) reduce to the initial values given by Eqs. (31). Furthermore, since $v=V_{t}$, we can integrate and find an exact solution to Laplace's equation for purposes of comparison with the difference solution. We obtain

$$
V=\frac{1}{2} \ln \left[(1+x)^{2}+t^{2}\right]
$$




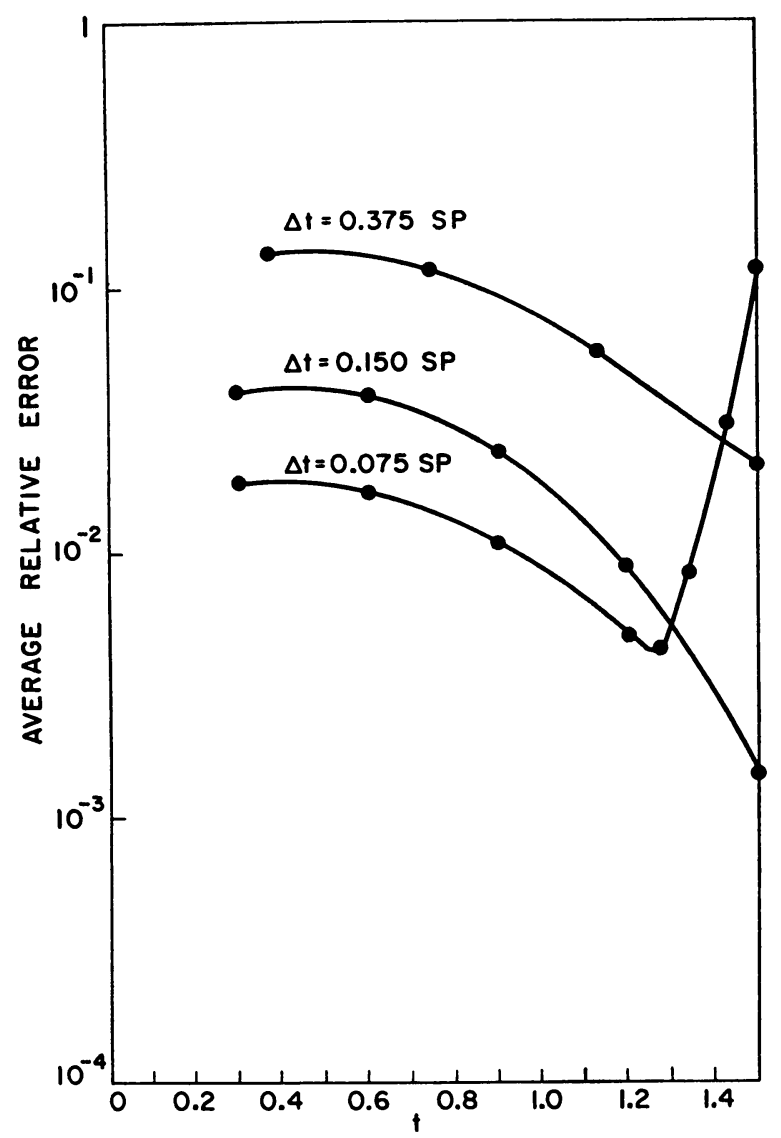

FIG. 5. Average relative error as a function of $t$ for $\mu=1 / 3$; a pole in the initial values lies at $x+$ $i t=-1$ and the base of the triangle of the finite difference mesh system lies between $0<x<1$.

By using the triangular mesh of Fig. 1 and the difference equations (24) through (26), values of $V$ were computed on a Burroughs 220 computer. The results were compared with those of the exact solution given by Eq. (33), and the average relative errors for each row of the triangular region were obtained. Figure 4 contains some representative results obtained by reducing $\Delta x=\Delta t$ from .125 to .003125 , and using 8 or 17 significant figures (single or double precision, respectively) for the difference solutions. It can be observed that a reduction in mesh size results in a reduction of the errors obtained, or the solution to the difference system converges to the solution of the differential equation, as long as $\Delta t$ in Eq. (30) is large enough to keep the exponentially growing error insignificant with respect to the normal truncation error. When $\Delta t$ is made small enough, this condition is violated, and the exponential error growth represented by the straight lines in Fig. 4 makes its appearance. When single precision computations did not allow further reduction of the mesh size, a change to double precision enabled us to carry out such a reduction until the exponential error growth appeared again. In the straight line regions of Fig. 4, relative errors can be represented by

$$
V=C e^{\alpha t} .
$$


The values of $\alpha$ predicted by Eq. (30), indicated in the figure, correspond to the exponential growth observed in Fig. 4 almost exactly. The coefficient $C$ obtained in the computations is of the order of $10^{-10}$ for single precision and $10^{-20}$ for double precision. It is observed that these coefficients are much smaller than one unit in the least significant digit used in the computation. Any round-off error in the computation is due mainly to the Fourier term with $\beta=\pi / 4 \Delta x$. By assuming random errors on the base line, one would expect this component, and therefore, $C$, above, to have an initial amplitude far less than the estimate of one unit in the least significant digit. Clearly, this estimate is quite conservative. However, due to the large value of $\alpha$ in the exponential, it is possible to determine when the exponential error growth will set in with reasonable accuracy.

Although the conditions for convergence given in Section 2a, are only sufficient, it appears interesting to observe what happens numerically when they are violated. For this purpose, a solution to the last problem was repeated with a mesh such that $\Delta t=3 \Delta x$, in two regions:

$$
0 \leq x \leq 1 \text { and }-.95 \leq x \leq 0.05
$$

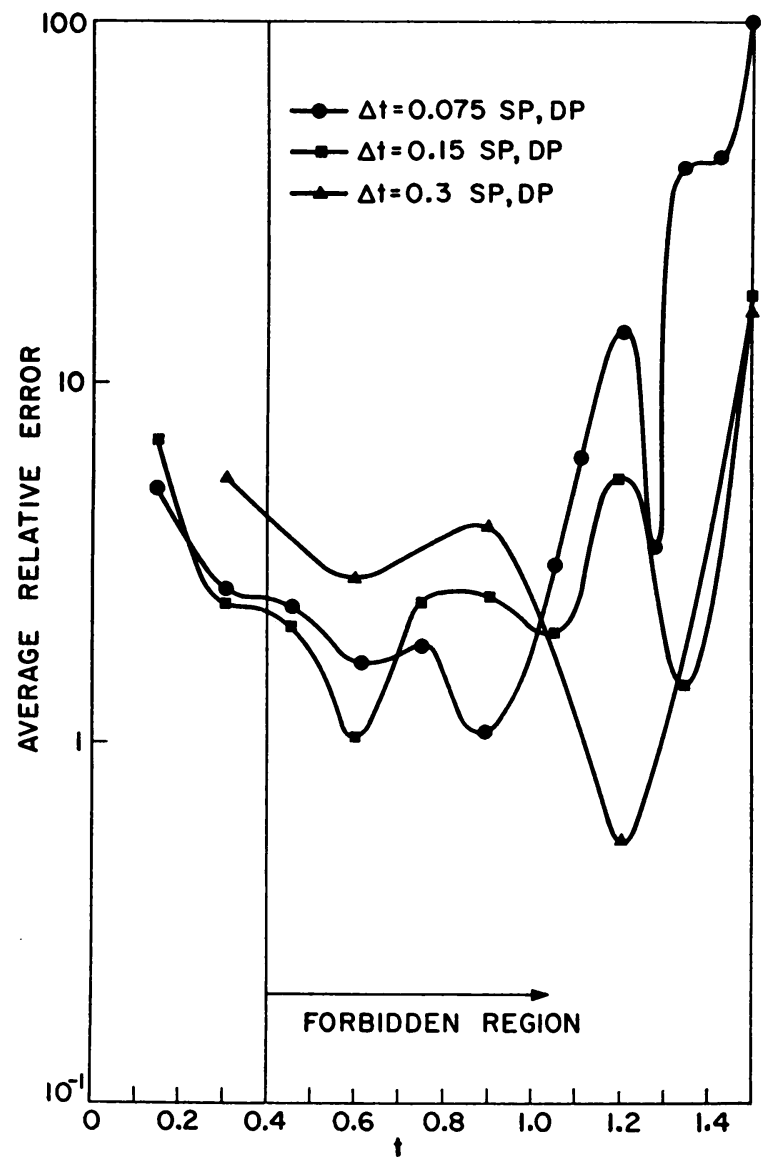

Fig. 6. Average relative error as a function of $t$ for $\mu=1 / 3$. A pole in the initial values lies at $x+$ $i t=-1$ and the base of the triangle of the finite difference mesh lies between $-.95<x<.05$. 
The first region was used as a control, since convergence was to be expected in it. In the second region, however, we were attempting to carry the difference solution into the "shadow" of the singularity at $(x, t)=(-1,0)$. The results show that, while for $0 \leq x \leq 1$ convergence is obtained as in the case of $\Delta x=\Delta t$ (see Fig. 5) no convergence is obtained in the second case (Fig. 6). Moreover, the ill behavior of the results is not affected by the number of digits used in the computation. These results are a good confirmation of the theory developed above.

4. Three-dimensional problem in cylindrical coordinates. Results of computation indicate very clearly that convergence and stability in the solution of Laplace's equation as an initial value problem in other mesh or coordinate systems behave very much as in the case studied above. Of particular importance is the solution of the three-dimensional, axially symmetric system of Laplace's equations in curvilinear coordinates:

$$
V_{u u}+V_{s s}+\frac{1}{r} r_{s} V_{s}+\frac{1}{r} r_{u} V_{u}=0, \quad r_{u u}+r_{s s}=0,
$$

with analytic initial values given along an arc (See Fig. 7).
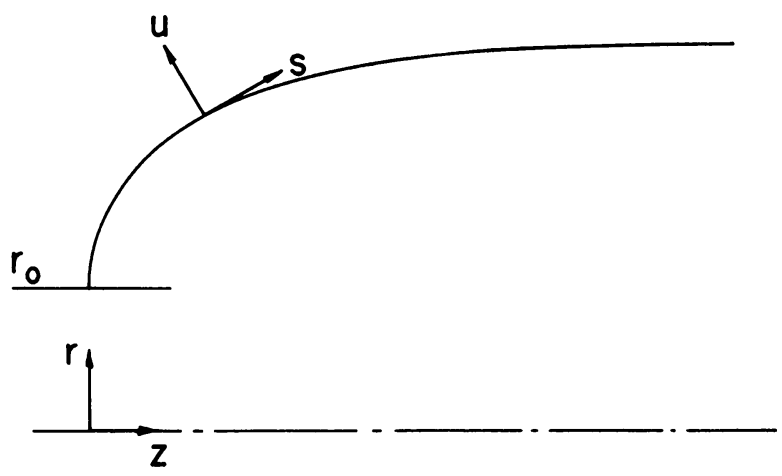

Fig. 7. Boundary curve on which initial values are given for the solution of Laplace's equation in an axially symmetric system

The difference equations used for this problem as obtained from Eq. (35) are:

$$
\begin{aligned}
& \frac{2}{(\Delta u)^{2}}[V(u+\Delta u, s)-2 V(u, s)+V(u-\Delta u, s)] \\
& +\frac{2}{(\Delta s)^{2}}[V(u, s+\Delta s)-2 V(u, s)+V(u, s-\Delta s)] \\
& +\frac{1}{r(u, s)}\left[\frac{r(u, s+\Delta s)-r(u, s-\Delta s)}{2 \Delta s}\right]\left[\frac{V(u, s+\Delta s)-V(u, s-\Delta s)}{2 \Delta s}\right] \\
& \quad+\frac{1}{r(u, s)}\left[\frac{r(u+\Delta u, s)-r(u-\Delta u, s)}{2 \Delta u}\right]\left[\frac{V(u+\Delta u, s)-V(u-\Delta u, s)}{2 \Delta u}\right]=0 . \\
& \frac{r(u+\Delta u, s)-2 r(u, s)+r(u-\Delta u, s)}{(\Delta u)^{2}}+\frac{r(u, s+\Delta s)-2 r(u, s)+r(u, s-\Delta s)}{(\Delta s)^{2}}=0 .
\end{aligned}
$$

The computations were carried out in double precision on a Burroughs 220 computer and the results obtained for decreasing mesh size were compared to results obtained 
for the same problem by the method of analytic continuation [4]. Steady convergence toward the known solution was obtained, until the point was reached in which the mesh size was small enough to cause an exponential growth of errors, as expected.

\section{BIBLIOGRAPHY}

1. G. Dahlquist, Convergence and stability for a hyperbolic difference equation with analytic initial values, Math. Scand. 2, 91-102 (1954)

2. E. C. Titchmarsh, The theory of functions, Oxford University Press, England, 1939, Sect. 1.51

3. F. John, Continuous dependence on data for solutions of partial differential equations with a prescribed bound, Comm. on Pure and Appl. Math. 13, 551-585 (1960)

4. K. J. Harker, Determination of electrode shapes for axially symmetric electron guns, J. Appl. Phys. 31, 2165-2170 (1960) 